\title{
Prevalencia de malformaciones congénitas detectadas al nacimiento en un hospital de segundo nivel en Sinaloa
}

Calderón-Alvarado $\mathrm{AB}^{1}$, Rojas-Villegas $\mathrm{MS}^{2}$, Dehesa-López $\mathrm{E}^{3}$

Resumen

INTRODUCCIÓN: la prevalencia de malformaciones congénitas (MC) detectadas al nacimiento es de 2 a 5.5 neonatos vivos, variando significativamente según las características de la población y metodología del estudio.

OBJETIVO: determinar la prevalencia y tipos de $M C$ en los recién nacidos atendidos por el servicio de neonatología en un periodo de un año.

MATERIAL Y MÉTODOS: estudio transversal. Se revisaron expedientes clínicos de recién nacidos vivos atendidos en el servicio de neonatología de nuestra institución durante el año 2015. Se incluyeron neonatos con alteraciones morfológicas detectadas al nacimiento y/o antes del alta hospitalaria, referidas en el expediente.

RESULTADOS: se detectaron 117 MC en 98 neonatos, de un total de 4,097 estudiados, obteniendo una prevalencia de $2.39 \%$ en el análisis por individuo y de $2.85 \%$, tomando en cuenta el total de MC. Los aparatos y sistemas más afectados fueron: genitourinario (29.1\%), osteomuscular (21.4\%) y circulatorio (16.2\%). Las MC más frecuentes fueron: criptorquidia $(14.5 \%)$, hipospadias $(12.8 \%)$ y anquiloglosia (10.3\%).

CONCLUSIÓN: la tasa de prevalencia de MC fue de 23.9 por 1,000 recién nacidos vivos, lo cual coincide con la reportada en el resto del mundo, siendo el aparato genitourinario el más afectado y la criptorquidia la MC más frecuente en nuestra población.

PALABRAS CLAVE: malformaciones congénitas, criptorquidia.
${ }^{1}$ Departamento de Neonatología, Hospital Civil de Culiacán.

²Pediatra, Departamento de Neonatología.

${ }^{3}$ Subdirector de Investigación en el Centro de Investigación y Docencia en Ciencias de la Salud (CIDOCS), Hospital Civil de Culiacán.

Centro de Investigación y Docencia en Ciencias de la Salud (CIDOCS), Hospital Civil de Culiacán.

Recibido: 2 de diciembre del 2016

Aceptado: 13 de marzo del 2017

Correspondencia

Ana Beatriz Calderón Alvarado

anabe_calderon@hotmail.com

Este artículo debe citarse como

Calderón-Alvarado AB, Rojas-Villegas MS, DehesaLópez E. Prevalencia de malformaciones congénitas detectadas al nacimiento en un hospital de segundo nivel en Sinaloa. Acta Pediatr Mex. 2017;38(6):363-370. 


\section{Prevalence of congenital malformations detected at birth in a second level hospital.}

Calderón-Alvarado $\mathrm{AB}^{1}$, Rojas-Villegas $\mathrm{MS}^{2}$, Dehesa-López $\mathrm{E}^{3}$

\begin{abstract}
INTRODUCTION: The prevalence of congenital malformations (CM) detected at birth ranges between 2 and 5.5 live births, with significant variation, depending on the characteristics of the population and the study methodology.
\end{abstract}

OBJECTIVE: To determine the prevalence and type of CM in newborns attended by the neonatology service in a period of one year.

METHODS: Cross-sectional study. Clinical records of all live births attended in the neonatology service of our hospital during 2015 were reviewed. Neonates that were detected with morphological alterations at birth and/or before his discharge referred at the clinical record, were included.

RESULTS: We detected 117 congenital malformations in 98 neonates, from a total of 4,097 newborns studied, resulting in a prevalence of $2.39 \%$ in the analysis per individual and $2.85 \%$ when taking into account all malformations. The most commonly affected systems were: genitourinary (29.1\%), osteomuscular (21.4\%) and circulatory (16.2\%). The most frequent CM were cryptorchidism (14.5\%), hypospadias (12.8\%) andankyloglossia (10.3\%).

CONCLUSION: The prevalence rate of CM was 23.9 per 1,000 live newborns, which coincides with the reported in the rest of the world, being the genitourinary system the most affected one and the cryptorchidism the most frequent CM in our population.

KEYWORDS: congenital malformations; cryptorchidism
${ }^{1}$ Departamento de Neonatología, Hospital Civil de Culiacán.

${ }^{2}$ Pediatra, Departamento de Neonatología. ${ }^{3}$ Subdirector de Investigación en el Centro de Investigación y Docencia en Ciencias de la Salud (CIDOCS), Hospital Civil de Culiacán.

Centro de Investigación y Docencia en Ciencias de la Salud (CIDOCS), Hospital Civil de Culiacán.

Correspondence

Ana Beatriz Calderón Alvarado anabe_calderon@hotmail.com

\section{INTRODUCCIÓN}

En la actualidad, los términos: defectos de nacimiento, malformaciones y anomalías congénitas se usan de forma indistinta para describir todas aquellas alteraciones estructurales o funcio- nales, que se producen durante el desarrollo intrauterino y que están presentes al nacimiento, detectadas en ese momento o posteriormente. ${ }^{1}$

Estudios publicados alrededor del mundo reportan una prevalencia de malformaciones 
congénitas al nacimiento entre 20 y 55 casos por cada 1000 neonatos vivos, con una variación significativa, dependiendo de las características demográficas de la población estudiada, la metodología y los criterios diagnósticos utilizados. ${ }^{2,3}$

Las malformaciones congénitas (MC) son responsables directos o indirectos de una alta proporción de muertes perinatales e infantiles, ubicándose como la segunda causa de muerte en menores de 1 año en nuestro país, ${ }^{4,5}$ además de las secuelas físicas, repercusiones emocionales y económicas que éstas conllevan. ${ }^{6}$

En México y otros países de América Latina las malformaciones congénitas se encuentran dentro de las principales causas de morbilidad y mortalidad en la infancia, por lo que un diagnóstico temprano resulta de gran importancia para brindar un manejo oportuno de las mismas. Por otro lado, es necesaria la creación y retroalimentación de registros locales y nacionales de estas patologías, que permitan conocer su frecuencia de presentación y características clínicas en nuestro medio, para una mejor vigilancia epidemiológica de estas patologías.

\section{OBJETIVO}

Determinar la prevalencia y el tipo de malformaciones congénitas más frecuentes en los recién nacidos atendidos por el servicio de Neonatología de nuestra institución.

\section{MATERIAL Y MÉTODOS}

Se realizó un estudio trasversal en el que se revisaron todos los expedientes clínicos de los recién nacidos vivos, atendidos en el servicio de neonatología de nuestro hospital, en el periodo entre el 01 de enero al 31 de diciembre del año 2015.
El diagnóstico de MC fue admitido si en el expediente clínico el médico neonatólogo o pediatra, que realizó la valoración del recién nacido, especificaba la presencia de una o más alteraciones morfológicas internas o externas detectadas al nacimiento (nota inicial) y/o antes de su egreso hospitalario (nota de egreso).

Se consideraron las malformaciones congénitas mencionadas en el Capítulo XVII de la décima revisión de la Clasificación Estadística Internacional de Enfermedades y Problemas de Salud Conexos (CIE-10), dedicado a las «malformaciones congénitas, deformaciones y anomalías cromosómicas», excluyendo a los errores innatos del metabolismo o los trastornos hematológicos de origen prenatal. El diagnóstico final de las malformaciones congénitas se integró de acuerdo al juicio clínico final del pediatra o neonatólogo que valoró al recién nacido, y en el caso de cromosomopatía, ésta fue confirmada por cariotipo.

Se analizó la frecuencia por individuo y por número de malformaciones. Se recabaron además variables perinatales: género, peso corporal al nacer, edad gestacional por fecha de última menstruación, peso para edad gestacional (clasificación según Battaglia y Lubchenco) y motivo de egreso en cada recién nacido); y maternos: edad, lugar de residencia, escolaridad, nivel socioeconómico, número de gestaciones, antecedente de aborto, control prenatal, ingesta de ácido fólico, toxicomanías, y comorbilidades.

Se utilizó estadística descriptiva con medidas de tendencia central y dispersión de los datos a base de medias y desviaciones estándar, en el caso de variables continuas y con frecuencias y proporciones en el caso de variables categóricas. Los datos fueron analizados con el paquete estadístico SPSS versión 23. 


\section{RESULTADOS}

\section{Características generales de la población}

\subsection{Maternas}

La edad promedio fue de $25 \pm 6$ años. El $84.7 \%$ $(\mathrm{n}=83)$ provenían de un medio socioeconómico bajo, el $51 \%(n=50)$ tenía al menos un embarazo previo y el $15.3 \%(n=15)$ tenía el antecedente de aborto. El $89.8 \%(n=88)$ refirieron haber acudido a control prenatal y el 100\% refirió haber consumido ácido fólico durante el embarazo. La comorbilidad más frecuentemente observada fue la enfermedad hipertensiva del embarazo con $71.4 \%(n=10)$ de los casos. El resto de las características maternas de nuestra población se muestran en la Cuadro 1.

\subsection{Del recién nacido}

El $65.3 \%(n=64)$ de los recién nacidos correspondían al sexo masculino, con una proporción de afección por sexo (masculino:femenino) de 1.88 , lo que indica una mayor prevalencia de malformaciones congénitas en neonatos masculinos. La media de peso fue $3,296 \pm 517$ gramos. El $81.6 \%$ de los recién nacidos presentó un peso "adecuado para su edad gestacional", sin encontrar recién nacidos "pequeños para la edad gestacional" en nuestra población. Por otro lado, el $85.7 \%$ ( $n=84)$ de los casos fueron neonatos a término. El resto de las características de los recién nacidos están puntualizadas en la

\section{Cuadro 2.}

\section{Prevalencia de malformaciones congénitas}

Durante el periodo de tiempo estudiado se atendieron un total de 4,417 nacidos vivos en nuestro hospital, de los cuales 4,097 (92.8\%) fueron incluidos en el estudio y 320 (7.2\%) fueron excluidos por falta de información en el expediente clínico.
Cuadro 1. Características demográficas y gineco-obstétricas maternas

\begin{tabular}{|c|c|c|}
\hline Variable & Frecuencia & Porcentaje \\
\hline \multicolumn{3}{|l|}{ Edad } \\
\hline$\leq 19$ años & 18 & 18.4 \\
\hline 20-34 años & 72 & 73.5 \\
\hline$\geq 35$ años & 8 & 8.2 \\
\hline \multicolumn{3}{|l|}{ Escolaridad } \\
\hline Ninguna & 2 & 2.0 \\
\hline Primaria & 16 & 16.3 \\
\hline Secundaria & 34 & 34.7 \\
\hline Bachillerato & 27 & 27.6 \\
\hline Licenciatura o mayor & 19 & 19.4 \\
\hline \multicolumn{3}{|l|}{ Nivel socioeconómico } \\
\hline Medio-bajo & 83 & 84.7 \\
\hline Medio & 12 & 13.3 \\
\hline Bajo & 2 & 2.0 \\
\hline \multicolumn{3}{|l|}{ Gesta } \\
\hline Multigesta & 50 & 51 \\
\hline Primigesta & 48 & 49 \\
\hline Antecedente de aborto & 15 & 15.3 \\
\hline $\begin{array}{l}\text { Control prenatal adecuado } \\
(\geq 5 \text { consultas })\end{array}$ & 88 & 89.8 \\
\hline $\begin{array}{l}\text { Ingesta de ácido fólico durante } \\
\text { el embarazo }\end{array}$ & 98 & 100 \\
\hline Toxicomanías & 2 & 2 \\
\hline Enfermedad materna & 14 & 14.3 \\
\hline $\begin{array}{l}\text { Enfermedad hipertensiva del } \\
\text { embarazo }\end{array}$ & 10 & $71.4 \%$ \\
\hline - Hipertensión gestacional & 6 & $42.9 \%$ \\
\hline - Preeclampsia & 2 & $14.3 \%$ \\
\hline - Eclampsia & 1 & $7.1 \%$ \\
\hline $\begin{array}{l}\text { - Hipertensión arterial } \\
\text { crónica }\end{array}$ & 1 & $7.1 \%$ \\
\hline Diabetes gestacional & 2 & $14.3 \%$ \\
\hline Acretismo placentario & 1 & $7.1 \%$ \\
\hline Hipotiroidismo & 1 & $7.1 \%$ \\
\hline
\end{tabular}

Se documentaron malformaciones en 98 recién nacidos vivos, para una prevalencia del $2.39 \%$ en nuestra población. De éstas, el $4.1 \%(n=4)$ correspondieron a malformaciones congénitas sindrómicas y $95.9 \%(n=94)$ a malformaciones no sindrómicas, 84 aisladas y 10 múltiples. 
Cuadro 2. Características perinatales

\begin{tabular}{|c|c|c|}
\hline Variable & Frecuencia & Porcentaje \\
\hline \multicolumn{3}{|l|}{ Sexo } \\
\hline $\begin{array}{l}\text { Femenino } \\
\text { Masculino }\end{array}$ & $\begin{array}{l}34 \\
64\end{array}$ & $\begin{array}{l}34.7 \\
65.3\end{array}$ \\
\hline \multicolumn{3}{|l|}{ Edad gestacional (Capurro) } \\
\hline $\begin{array}{l}\text { Pretérmino (<37 sdg) } \\
\text { Término }(37-41 \mathrm{sdg}) \\
\text { Postérmino (>41 sdg) }\end{array}$ & $\begin{array}{c}10 \\
84 \\
4\end{array}$ & $\begin{array}{c}10.2 \\
85.7 \\
4.1\end{array}$ \\
\hline \multicolumn{3}{|c|}{ Peso para edad gestacional (Battaglia, Lubchenco) } \\
\hline $\begin{array}{l}\text { PEG (pequeño para edad) } \\
\text { APG (adecuado para edad } \\
\text { gestacional) }\end{array}$ & $\begin{array}{c}0 \\
80\end{array}$ & $\begin{array}{c}0 \\
81.6\end{array}$ \\
\hline $\begin{array}{l}\text { GEG (grande para edad } \\
\text { gestacional) }\end{array}$ & 18 & 18.4 \\
\hline
\end{tabular}

\section{Tipos de malformaciones congénitas}

En nuestra población se registraron un total de $117 \mathrm{MC}$, siendo la más frecuente la criptorquidia con $14.5 \%(n=17)$, seguida de hipospadias con $12.8 \%(n=15)$, anquiloglosia con $10.3 \%$ $(n=12)$, apéndice preauricular con $5.1 \%(n=6)$ $y$ hemangioma y sindactilia cada una con $4.3 \%$ $(\mathrm{n}=5)$. La totalidad de las MC encontradas en nuestra población y sus frecuencias observadas, se muestran en el Cuadro 3.

\section{Distribución de las MC por aparatos y sistemas}

En nuestra población, los aparatos y sistemas más afectados por MC fueron: el genitourinario con $29.1 \%(n=34)$, osteomuscular con $21.4 \%$ $(\mathrm{n}=25)$ y el sistema circulatorio con $16.2 \%(\mathrm{n}=$ 19) de los casos. El resto de aparatos y sistemas se muestra en el Cuadro 3.

\section{Evolución clínica de los recién nacidos con MC}

En relación a la evolución clínica de los recién nacidos con algún tipo de MC, el 90.8\% ( $\mathrm{n}=89)$ fue egresado por mejoría clínica; $6.1 \%(n=6)$ fueron trasladados para manejo quirúrgico o por
Cuadro 3. Frecuencia de malformaciones (clasificación por sistemas CIE-10) (continúa en la siguiente página)

\begin{tabular}{|c|c|c|c|}
\hline $\begin{array}{l}\text { Malformación } \\
\text { congénita }\end{array}$ & Frecuencia & Porcentaje & $\begin{array}{c}\text { Tasa por } \\
1,000 \mathrm{rn}^{*} \\
\text { vivos }\end{array}$ \\
\hline $\begin{array}{l}\text { Sistema nervioso } \\
\text { central }\end{array}$ & 1 & 0.9 & 0.24 \\
\hline Espina bífida & 1 & 0.9 & 0.24 \\
\hline Ojos cara y cuello & 13 & 11.1 & 3.17 \\
\hline $\begin{array}{l}\text { Apéndice } \\
\text { preauricular }\end{array}$ & 6 & 5.1 & 1.46 \\
\hline Microftalmia & 2 & 1.7 & 0.49 \\
\hline Blefarofimosis & 1 & 0.9 & 0.24 \\
\hline $\begin{array}{l}\text { Estenosis de vía } \\
\text { lagrimal }\end{array}$ & 1 & 0.9 & 0.24 \\
\hline $\begin{array}{l}\text { Lóbulo } \\
\text { supernumerario }\end{array}$ & 1 & 0.9 & 0.24 \\
\hline $\begin{array}{l}\text { Tabique nasal } \\
\text { desviado }\end{array}$ & 1 & 0.9 & 0.24 \\
\hline Vestigio braquial & 1 & 0.9 & 0.24 \\
\hline $\begin{array}{l}\text { Aparato } \\
\text { circulatorio }\end{array}$ & 19 & 16.2 & 4.6 \\
\hline $\begin{array}{l}\text { Persistencia de } \\
\text { conducto arterioso }\end{array}$ & 4 & 3.5 & 0.98 \\
\hline $\begin{array}{l}\text { Comunicación } \\
\text { interventricular }\end{array}$ & 3 & 2.6 & 0.73 \\
\hline $\begin{array}{l}\text { Estenosis de } \\
\text { válvula aórtica }\end{array}$ & 1 & 0.9 & 0.24 \\
\hline $\begin{array}{l}\text { Estenosis } \\
\text { subaórtica }\end{array}$ & 1 & 0.9 & 0.24 \\
\hline $\begin{array}{l}\text { Foramen oval } \\
\text { permeable }\end{array}$ & 1 & 0.9 & 0.24 \\
\hline Tetralogía de Fallot & 1 & 0.9 & 0.24 \\
\hline $\begin{array}{l}\text { Transposición de } \\
\text { grandes vasos }\end{array}$ & 1 & 0.9 & 0.24 \\
\hline $\begin{array}{l}\text { Túnel aorto } \\
\text { ventricular }\end{array}$ & 1 & 0.9 & 0.24 \\
\hline $\begin{array}{l}\text { Aparato } \\
\text { respiratorio }\end{array}$ & 1 & 0.9 & 0.24 \\
\hline $\begin{array}{l}\text { Laringomalacia } \\
\text { congénita }\end{array}$ & 1 & 0.9 & 0.24 \\
\hline $\begin{array}{l}\text { Labios, boca } \\
\text { y paladar }\end{array}$ & 16 & 13.7 & 3.9 \\
\hline Anquiloglosia & 12 & 10.3 & 2.93 \\
\hline $\begin{array}{l}\text { Fisura de } \\
\text { paladar duro }\end{array}$ & 2 & 1.7 & 0.49 \\
\hline Labio leporino & 2 & 1.7 & 0.49 \\
\hline
\end{tabular}


Cuadro 3. Frecuencia de malformaciones (clasificación por sistemas CIE-10) (continuación)

\begin{tabular}{|c|c|c|c|}
\hline $\begin{array}{l}\text { Malformación } \\
\text { congénita }\end{array}$ & Frecuencia & Porcentaje & $\begin{array}{c}\text { Tasa por } \\
1,000 \mathrm{rn}^{*} \\
\text { vivos }\end{array}$ \\
\hline Tracto digestivo & 1 & 0.9 & 0.24 \\
\hline Ano imperforado & 1 & 0.9 & 0.24 \\
\hline Órganos genitales & 34 & 29.1 & 8.30 \\
\hline Criptorquidia & 17 & 14.5 & 4.15 \\
\hline Hipospadias & 15 & 12.8 & 3.66 \\
\hline Epispadias & 2 & 1.7 & 0.49 \\
\hline $\begin{array}{l}\text { Sistema } \\
\text { osteomuscular }\end{array}$ & 25 & 21.4 & 6.10 \\
\hline Sindactilia & 5 & 4.3 & 1.22 \\
\hline Polidactilia & 4 & 3.4 & 0.98 \\
\hline Pie equino varo & 3 & 2.6 & 0.73 \\
\hline $\begin{array}{l}\text { Artrogriposis } \\
\text { múltiple congénita }\end{array}$ & 2 & 1.7 & 0.49 \\
\hline $\begin{array}{l}\text { Luxación congénita } \\
\text { de cadera }\end{array}$ & 2 & 1.7 & 0.49 \\
\hline Agenesia de dedos & 1 & 0.9 & 0.24 \\
\hline $\begin{array}{l}\text { Deformidad congé- } \\
\text { nita de la mano }\end{array}$ & 1 & 0.9 & 0.24 \\
\hline $\begin{array}{l}\text { Deformidad congé- } \\
\text { nita de los pies }\end{array}$ & 1 & 0.9 & 0.24 \\
\hline Gastrosquisis & 1 & 0.9 & 0.24 \\
\hline $\begin{array}{l}\text { Hipoplasia de } \\
\text { mandíbula }\end{array}$ & 1 & 0.9 & 0.24 \\
\hline $\begin{array}{l}\text { Luxación congénita } \\
\text { de rodilla }\end{array}$ & 1 & 0.9 & 0.24 \\
\hline Pie equino valgo & 1 & 0.9 & 0.24 \\
\hline $\begin{array}{l}\text { Reducción longitu- } \\
\text { dinal de radio }\end{array}$ & 1 & 0.9 & 0.24 \\
\hline Talus vertical & 1 & 0.9 & 0.24 \\
\hline $\begin{array}{l}\text { Otras } \\
\text { malformaciones }\end{array}$ & 3 & 2.6 & 0.73 \\
\hline $\begin{array}{l}\text { Pezón } \\
\text { supernumerario }\end{array}$ & 2 & 1.7 & 0.49 \\
\hline $\begin{array}{l}\text { Aplasia dérmica } \\
\text { congénita }\end{array}$ & 1 & 0.9 & 0.24 \\
\hline $\begin{array}{l}\text { Anormalidades } \\
\text { cromosómicas }\end{array}$ & 4 & 3.4 & 0.97 \\
\hline Síndrome Down & 3 & 2.6 & 0.73 \\
\hline $\begin{array}{l}\text { Síndrome de } \\
\text { Edwards }\end{array}$ & 1 & 0.9 & 0.24 \\
\hline
\end{tabular}

*Recién nacidos. estancia prolongada a otra institución y el 3.1\% $(n=3)$ falleció por una causa directa o indirectamente relacionada a la MC.

\section{DISCUSIÓN}

En nuestra población la prevalencia de MC por individuo afectado fue de $2.4 \%$ y por número de malformaciones fue de $2.8 \%$. Esta prevalencia se encuentra dentro del rango referido en la literatura a nivel internacional, la cual varía dependiendo de la región analizada y los criterios de inclusión y diagnóstico utilizados en cada estudio.

En relación al tipo de $\mathrm{MC}$ y su distribución por aparatos y sistemas, en nuestra población el sistema genitourinario fue el más afectado, siendo la criptorquidia la MC más frecuente con una prevalencia de $0.4 \%$. Estos hallazgos contrastan con los resultados reportados en otras series en el estado de Sinaloa. En el 2009, Ramos Parra y cols. reportaron una prevalencia de MC en recién nacidos en el Hospital de la Mujer de Sinaloa de $0.95 \%$, siendo el aparato gastrointestinal el más afectado. Esta diferencia puede ser explicada por el hecho de que los autores incluyeron únicamente MC mayores. En este sentido, la prevalencia de MC mayores en nuestra población fue de $0.65 \%$, parecida a la reportada por Ramos y cols. ${ }^{7}$ Por otro lado, Flores Nava y cols. reportaron una prevalencia individual de MC de $1.8 \%$ y global de $2.9 \%$ en recién nacidos vivos o muertos en el Hospital Gea González de la Ciudad de México, siendo las MC del sistema músculo esquelético las más frecuentes, incluyendo al paladar hendido con o sin labio hendido en este grupo. ${ }^{6}$ Un dato interesante es que a pesar de que estos autores consideraron las MC observadas en nacidos vivos y muertes fetales, la prevalencia de MC por individuo fue mayor en nuestra población en la que únicamente incluimos nacidos vivos, pero fue menor en el número total de malformaciones. 
Según el INEGI las cifras de mortalidad infantil han ido descendiendo con el paso de los años, no así el número de muertes atribuidas a malformaciones congénitas, Ilegando a ocupar el segundo lugar como causa de muerte infantil en menores de un año. ${ }^{5}$ En nuestra población, el $3.1 \%(n=3)$ de los recién nacidos con alguna MC falleció por una causa directa o indirectamente relacionada a la MC. En este sentido, a pesar de que en los últimos años, muchos de los factores causales de MC han sido identificados, la mayoría de ellos permanecen desconocidos, lo que ha dificultado la prevención de las mismas. ${ }^{8}$

Por otra parte, Navarrete Hernández y cols. reportaron en 2013 una prevalencia de MC de $0.7 \%$ en los certificados de nacimiento de los nacidos vivos. Esta prevalencia muy por debajo de la reportada en otras series nacionales, se traduce en un subregistro de las MC en nuestro país, por lo que es necesaria la implementación de programas de validación y capacitación para fortalecer estos sistemas de registro. ${ }^{9}$

La mayoría de los recién nacidos afectados por MC en nuestra población fueron productos de embarazos de 37 a 41 semanas de gestación, lo que nos indica que se trata de MC que permiten un embarazo a término sin afectación del crecimiento intrauterino, ya que en su mayoría $(81.6 \%)$, los recién nacidos presentaron un peso adecuado para la edad gestacional. La mayor parte fueron de sexo masculino, tal como se ha reportado en otras descripciones.

La edad materna promedio fue de 25 años, sólo el $26.6 \%(n=26)$ de las madres se encontraban en un rango de edad considerado de alto riesgo ( $\leq 19$ y $\geq 35$ años) para MC, especialmente cromosómicas. En este rubro, en nuestra población se detectaron un caso de síndrome de Edwards (trisomía 18) y tres casos de síndrome Down (trisomía 21), que representa la cromosomopatía más frecuente en nacidos vivos. En tres de estos casos, la edad materna fue mayor de 35 años, que constituye uno de los principales factores de riesgo asociados con anomalías cromosómicas. ${ }^{10}$

Las MC de mayor frecuencia en nuestro estudio fueron las de órganos genitales, destacando la criptorquidia y el hipospadias, ambas consideradas de etiología multifactorial y para las cuales se han descritos diversos factores de riesgo: genéticos, antecedente familiar de dicha anomalía; gestacionales: tabaquismo, alcoholismo, diabetes gestacional, inseminación artificial, administración de estrógeno); perinatales: prematurez, bajo peso, gestación múltiple, presentación pélvica), entre otros. ${ }^{11}$

La prematurez fue el único factor de riesgo observado en nuestra población, la cual estuvo presente únicamente en 3 de 15 casos de hipospadias. Para ambas entidades la identificación oportuna es muy importante, ya que en el caso de la criptorquidia un retraso en el diagnóstico se asocia a infertilidad y cáncer testicular, y en el caso de el hipospadias porque afecta la calidad de vida del individuo. ${ }^{12}$

Nuestra investigación presenta ciertas debilidades, siendo la más importante y debido a la naturaleza transversal/retrolectiva del trabajo, la posibilidad de un subregistro de MC no referidas por el neonatólogo/pediatra en el expediente clínico o no detectadas por el investigador en el expediente clínico, además de la debilidad de nuestro trabajo para estudiar factores o exposiciones asociadas a MC, lo que limita la generación de hipótesis sólidas. Sin embargo, consideramos que dichas debilidades no invalidan nuestros resultados.

\section{CONCLUSIONES}

La prevalencia de MC por individuo fue de 2.39, lo cual se encuentra dentro del rango reportado en la literatura. El aparato genitourinario fue 
el más afectado y la criptorquidia la MC más frecuente en nuestra población. Nuestros resultados resaltan la importancia de establecer una vigilancia epidemiológica continua que permita un diagnóstico temprano y tratamiento oportuno de las MC; así como la importancia de la creación y retroalimentación de registros locales y nacionales que permitan conocer con mayor precisión las características epidemiológicas y clínicas de las MC en nuestro medio. Finalmente, nuestros resultados pueden sentar las bases para futuras investigaciones que permitan conocer los factores de riesgo, pronóstico, calidad de vida, etc... de recién nacidos con MC.

\section{Conflicto de intereses CI POLICY}

Los hallazgos, opiniones y puntos de vista contenidos en la investigación original "Prevalencia de malformaciones congénitas detectadas al nacimiento en un hospital de segundo nivel en Sinaloa" son particulares y no resultado de una posición oficial del Hospital Civil de Culiacán, el Centro de Investigación en Ciencias de la Salud o la Universidad Autónoma de Sinaloa.

\section{REFERENCIAS}

1. World Health Organization. Congenital anomalies. Factsheet $\mathrm{N}^{\circ} 370$. October 2012. Disponible en: http://www. who.int/mediacentre/factsheets/fs370/en/index.html (Último acceso: enero 2016).

2. Cragan JD, Khoury MJ. Effect of prenatal diagnosis on epidemiologic studies of birth defects. Epidemiology. 2000;11:695-9.
3. Clayton-Smith J, Donnai D. Human malformations. En: Rimoin DL, Connor JM, Pyeritz RE, Korf BR, eds. Emery and Rimoin's Principles and Practice of Medical Genetics. Vol. 1. New York: Churchill Livingstone; 2012. pp. 488-500.

4. Fernández-Cantón SB, Gutiérrez-Trujillo G, ViguriUribe R. Principales causas de mortalidad infantil en México: tendencias Recientes. Bol Med Hosp Infant Mex 2012;69(2):144-148.

5. INEGI. Estadística/Registros Administrativos/Mortalidad: Mortalidad General. Consulta en línea. http://www.inegi. org.mx/sistemas/olap/Proyectos/bd/continuas/mortali$\mathrm{dad} /$ MortalidadGeneral.asp?s=est\&c=11144\&proy=mort mg (Último acceso 10 de enero 2015).

6. Flores-Nava G, Pérez-Aguilera TV, Pérez-Bernabé MM. Malformaciones congénitas diagnosticadas en un hospital general. Revisión de cuatro años. Acta Pediatr Mex. 2011;32(2):101-106

7. Ramos-Parra E, Monzon-Ruelas A, Dautt-Leyva JG. Frecuencia de Malformaciones Congénitas en Recién Nacidos del Hospital de la Mujer. Arch Salud Sin. 2011;5(4):101-105.

8. Werler MM. Congenital Malformations and Consequential Epidemiology. Curr Epidemiol Rep. 2015;2:8-12.

9. Navarrete-Hernández R, Canún-Serrano $S$, Reyes-Pablo AE, Sierra-Romero MC, Valdés-Hernández J. Prevalencia de malformaciones congénitas registradas en el certificado de nacimiento y de muerte fetal. México, 2009-2010. Bol Med Hosp Infant Mex 2013;70(6):499-505.

10. Sirerra-Romero MC, Navarrete-Hernández E, Canún-Serrano S, Reyes-Pablo AE, Valdés-Hernández J. Prevalencia del síndrome de Down en México utilizando los certificados de nacimiento vivo y de muerte fetal durante el periodo 2008-2011. Bol Med Hosp Infant Mex. 2014;71(5):292-297.

11. Egbe A, Uppu S, Lee S, Stroustup A, Ho D, Srivastava S. Congenital Malformations in the Newborn Population: A Population Study and Analysis of the Effect of Sex and Prematurity. Pediatrics and Neonatology. 2015;56:25-30.

12. López-Cruz G, Pérez-Campos E, Hernandez-Cruz P. Criptorquidia: Importancia del Diagnóstico Oportuno. Bol Clin Hosp Infant. Edo Son. 2007;24(1):32-37. 